Title: Network Energy Consumption Assessment of Conventional Mobile Services and Over-the-top Instant Messaging Applications

\title{
Author list
}

Ming Yan ${ }^{1,2}$, Chien Aun Chan ${ }^{1}$, Wenwen $\mathrm{Li}^{3}$, Chih-Lin I ${ }^{3}$, Sen Bian ${ }^{3}$, André F. Gygax ${ }^{1,4}$, Christopher Leckie $^{1,5}$, Kerry Hinton ${ }^{1}$, Elaine Wong ${ }^{1}$, Ampalavanapillai Nirmalathas ${ }^{1}$

\section{Affiliations}

${ }^{1}$ Centre for Energy-Efficient Telecommunications (CEET), The University of Melbourne, 3010, Victoria, Australia.

${ }^{2}$ Faculty of Science and Technology, Communication University of China, Beijing, 100024, China.

${ }^{3}$ Green Communications Research Center (GCRC), China Mobile Research Institute, Beijing, 100053, China.

${ }^{4}$ Department of Finance, The University of Melbourne, 3010, Victoria, Australia.

${ }^{5}$ Department of Computing and Information Systems, The University of Melbourne, 3010, Victoria, Australia.

\begin{abstract}
The rapid growth in the energy consumption of mobile networks has become a major concern for mobile operators. Today's mobile networks' usage is dominated by over-the-top (OTT) applications and operators are keen to determine the network energy consumed by these OTT applications. With a recent shift in user behavior towards a preference for instant messaging (IM) applications over conventional mobile services, operators are interested in exploring what impact OTT IM applications such as WeChat will have on the energy consumption of a network when compared to a corresponding conventional mobile service.
\end{abstract}


Here, we present for the first time energy assessment models for mobile services based on real network and service measurements to address this need. Using WeChat as an OTT IM application example, our results show that WeChat consumes more network energy than conventional mobile services for both light users and heavy text users due to the network signaling energy overhead. In comparison, for heavy voice users, WeChat consumes less network energy since voice messages are first recorded and then sent in packet bursts. Our findings provide a quantitative analysis of the energy consumption of mobile services, which should be valuable for mobile operators and OTT application developers to improve the energy-efficiency of mobile applications and services.

Keywords: Energy efficiency; mobile services; OTT applications; mobile networks; energy assessment.

\section{Introduction}

Over recent years, the energy consumption of mobile networks has increased dramatically due to the rapid deployment of mobile base stations to accommodate the massive growth in mobile data traffic. For example, the total network energy consumption of China Mobile increased from 2,000 GWh in 2005 to $17,110 \mathrm{GWh}$ in 2014 [1], which is equivalent to about $14 \%$ of the total electricity generated by the 26 nuclear power plants (as at September 2015) currently operating in China [2]. The rapid growth in mobile data traffic is expected to continue into the near future. As estimated by [3], the global mobile traffic is expected to increase by a factor of 10 between 2014 and 2019. This means that more base stations will be deployed in the network to accommodate the rapidly increasing mobile traffic. As a result, meeting the future energy demand of growing mobile networks will become a real concern for mobile operators worldwide [4]. 
For some mobile operators, network energy consumption is not only regarded as an environmental sustainability problem but also considered as a significant operational expenditure due to associated power bills [4]. With the dominance of data traffic in mobile networks, mobile operators are facing a so-called 'revenue gap' or a 'scissor effect' in which billed revenues are not keeping up with traffic growth $[5,6]$. One way to reduce this 'revenue gap' is to reduce network costs [5]. As mobile access networks consume over $60 \%$ of the total energy consumption of a mobile operator [4], reducing the energy consumption of mobile access networks is vital for operators. Hence, finding more energy-efficient solutions has become a major focus in mobile access network research. For example, an energy model for wireless access networks has been proposed by [7] which was then widely used to quantify the energy efficiency of various Long Term Evolution (LTE) networks [8, 9]. Various proposed techniques and solutions to reduce the energy consumption and to improve the energy efficiency of mobile networks have been studied [10, 11].

Since mobile networks are dominated by mobile data traffic generated by over-the-top (OTT) applications, mobile operators are keen to assess the energy consumed by their networks in delivering these OTT mobile applications. Of particular interest are those applications that have significant impact on revenues such as OTT instant messaging (IM) applications (e.g., WeChat, Whatsapp, Line, etc.) and that are growing dramatically [12]. However, existing research on the impact of OTT mobile applications has been limited to the utilization of network resources [13-16] and power consumption of mobile devices [13, 17]. For example, the impact of OTT applications on mobile network resources was first studied by $[13,14]$. Both studies found that the 'heartbeat' mechanism of social networking OTT applications, which is used to send short packets to the server periodically to maintain an 'always-on' status, causes excessive signaling overhead in LTE networks due to the frequent establishment of radio resource control (RRC) connections [13, 14]. With hundreds of 
millions of smartphones sending frequent heartbeats, the signaling resources of mobile access networks are constantly highly utilized. Once network congestion occurs, the OTT applications will retry connections and cause a so-called signaling storm [15]. Several potential solutions were proposed to address the excessive signaling overhead caused by OTT IM applications [15, 16]. The authors of [16] proposed a new RRC 'keep-alive' state in addition to the RRC connected and idle states to reduce the overhead caused by the frequent establishment of RRC connections between the mobile device and the network. Furthermore, the authors of [15] proposed the use of a push server to manage all the 'heartbeat' messages from the same mobile device with the aim to reduce the total number of 'heartbeat' messages per device. In terms of power savings of mobile devices, the authors from [13] and [17] have proposed several techniques that can be used to reduce the power consumption of OTT applications in mobile devices.

However, the above studies focus only on reducing the energy consumption of the mobile network, quantifying the impacts of OTT IM applications on network resources, and reducing the power consumption of mobile devices. There is little work on fundamentally understanding how OTT IM mobile services and applications affect a network's energy consumption. Having similar functions as conventional mobile services, OTT IM applications have emerged as substitutes for conventional mobile services such as short messaging service (SMS), multimedia messaging service (MMS) and voice call [18]. This shift in user behavior has had a serious impact on the revenues of mobile operators for whom a large portion of revenues is generated by conventional mobile services. For example, according to China Mobile's quarterly report, the average SMS usage of the company has declined from 88.4 messages per user per month in 2013 to 59.7 messages per user per month in 2015 [1]. Similarly, the average voice call usage of China Mobile has declined from 488 minutes per user per month in 2013 to 420 minutes per user per month in 2015 [1]. 
A continuous decrease in revenues and an increase in network energy costs are problematic for mobile operators. In order to understand how mobile services affect the network energy consumption, we previously conducted a rigorous assessment of network energy consumption of multiple OTT applications such as text messaging services, Weibo, video play, file transfer protocol download, web browsing, etc. [19]. We found that the network energy consumption of mobile applications can vary based on various factors such as different base station cell sites and different user behavior at different times of the day. However, there is still a lack of in-depth research on the network energy efficiency of mobile services with similar functions, especially in comparing free-to-use OTT IM applications with conventional mobile services, which are the main revenue streams for mobile operators. In this paper, we address this gap in the literature by carrying out a thorough investigation on whether the use of an OTT IM application such as WeChat, which provides a service, e.g., a voice call or sending a text message, will be more energy-efficient when compared to a corresponding conventional mobile service. To achieve this, in Section 2 of the paper, we develop energy models to assess the energy consumption of delivering services in mobile wireless access networks by breaking the total network energy consumption into signaling and data energy components. This separation of energy consumption will highlight the difference in energy profiles of different mobile services. The value of this energy model lies in its ability to enable mobile operators to assess the energy consumption of any mobile services delivered through their mobile access network. Furthermore, the proposed energy models can be used in instances where assessing and reporting the carbon footprint of mobile services by the network operator is mandated. In Section 3, we use the energy models to compare the network energy consumption of WeChat services and conventional mobile services under different daily usage and user behavior scenarios. Our results show that WeChat consumes more network energy than conventional mobile services for light users 
with low daily usage due to a large amount of network signaling energy consumed by 'heartbeat' signals. Furthermore, our results show that WeChat consumes more network energy than SMS for heavy text message users due to excessive 'hint' signals, which are used to notify the recipient when a sender starts typing a message. For heavy voice message users, WeChat consumes less network energy compared to a conventional voice call because the voice message is first recorded and then sent in packet bursts by WeChat. In Section 4, we analyze the root cause of energy consumption of OTT IM services based on different user behaviors. We also discuss the potential solutions to reduce the overall network energy consumption of OTT IM services. Finally, a summary of the main findings reported in this paper is presented in Section 5.

\section{Network energy consumption model for mobile services}

Here, we present the energy model used to assess network energy consumption of mobile services in an LTE access network. Given the power consumption profile of a base station, our energy model allocates a proportion of the base station power consumption to each mobile service based on the data traffic and signaling traffic generated by the service. Therefore, understanding the power consumption profile of the base station used to serve the user is the first step in developing an energy model for mobile services.

\subsection{Overview of base station power profile}

Based on real measurements from the China Mobile Research Institute, Figure 1 plots the input power consumption of the base station versus the radio resource utilization. We observe that a linear relationship exists between the power consumption of a base station and the radio resource utilization (here, the radio resource utilization is the number of utilized physical resource blocks (PRB) [20]). The base station power consumption profile is 
consistent with measurements from [7] and [21]. This power profile consists of a baseline power $\left(P_{\text {base }}\right)$, which corresponds to the condition where the base station has no connected users and no service traffic. Under this condition, small levels of radio resources are used to transmit fixed signaling such as the cell reference signal (CRS), the primary synchronization signal (PSS), the secondary synchronization signal (SSS), the physical broadcast channel $(\mathrm{PBCH})$, the physical control format indicator channel, and the physical hybrid automatic repeat request indicator channel $(\mathrm{PHICH})$. As the utilization of radio resources increases, the power consumption of the base station will increase approximately linearly. At the maximum radio resource load, $R_{\max }$, the base station will reach a maximum power consumption of $P_{\max }$.

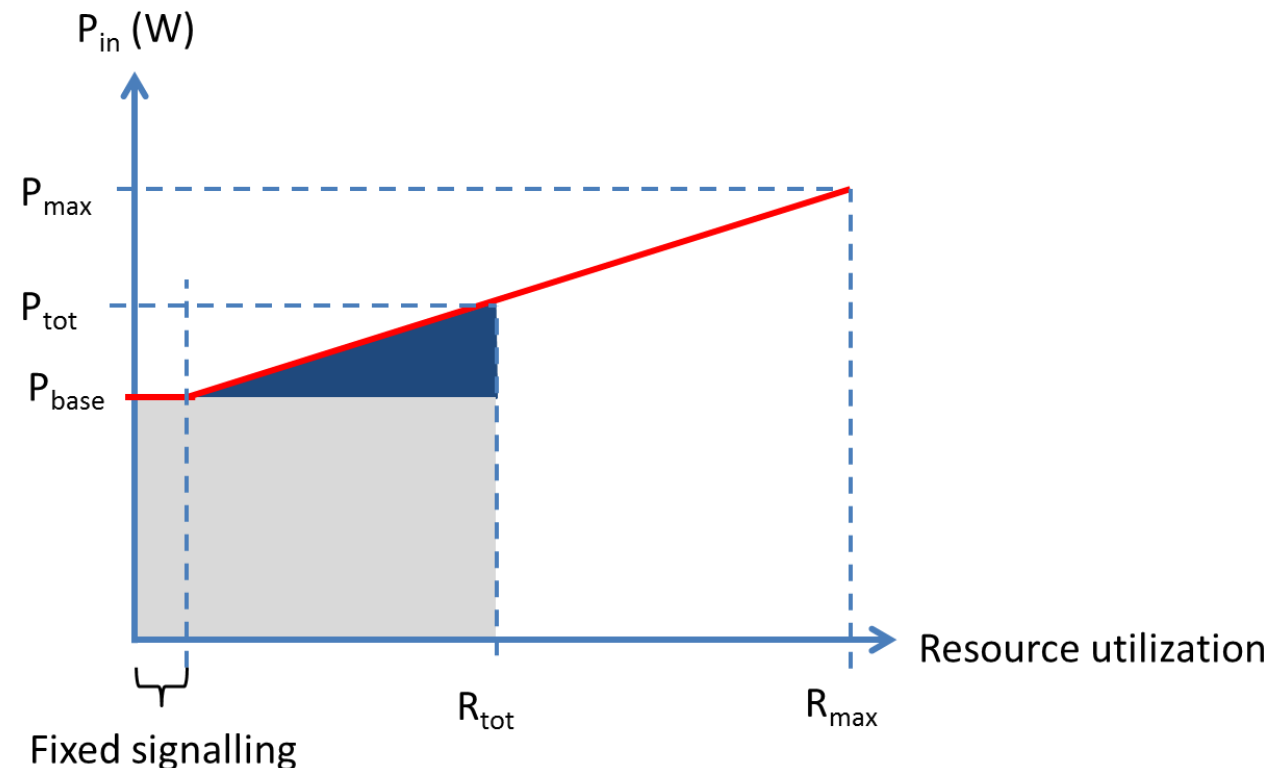

Figure 1. Power consumption of LTE macro base station as a function of radio resource utilization. The grey region represents the baseline power of the base station and the dark blue region represents the variable power of the base station.

For a given radio resource utilization at time $t, R_{t o t}(t)$, the total power consumption of the base station is $P_{t o t}(t)$. In general, multiple services are expected to share $R_{t o t}$ at time $t$. Hence, the purpose of the energy model described here is to allocate to each of those services a proportion of the base station power consumption at time $t$. It is important to note that this 
exercise is non-trivial due to the existence of signaling traffic overheads in mobile access networks. For a mobile device (e.g., a mobile phone) to establish and maintain a connection to the base station, a significant amount of signaling traffic is generated [22]. The energy model presented in this paper quantifies the energy consumption of both network data and network signaling traffic generated by the service.

There are three main components of a base station: the baseband unit (BBU); the radio remote unit (RRU); and the overhead (e.g., AC/DC and DC/DC conversion, cooling, etc.). Figure 2 shows the power consumption of the BBU as a function of radio resource utilization. Figure 3 shows the power consumption of the RRU as a function of radio resource utilization. Based on real measurements conducted in the China Mobile Research Institute on different BBUs and RRUs provided by different vendors, we found that the ratio $\mathrm{P}_{\text {base }} / \mathrm{P}_{\max }$ of the BBU is typically greater than $90 \%$. As for the RRU, the ratio of $\mathrm{P}_{\text {base }} / \mathrm{P}_{\max }$ could vary from $40 \%$ to $90 \%$ depending upon the type of base station (e.g., $40 \%$ for a macro base station and $90 \%$ for a femto base station given in [7]). $P_{R R U \_b a s e}$ comprises two components - the baseline power of the PA $\left(P_{P A_{-} b a s e}\right)$ and the power consumption of radio frequency $(\mathrm{RF})$ components $\left(P_{R F}\right)$ as shown in Figure 3. It should be noted that the power consumption values used in Section 3.1 to assess the network energy consumption of mobile services are based on the most commonly deployed BBU and RRU in the China Mobile network under the following configurations: 3-sector TD-LTE (S1/1/1) with 2 x 2 multi-input multi-output (MIMO) and bandwidth per sector of $20 \mathrm{MHz}$. However, different mobile operators at different countries use different configurations (e.g., frequency division-LTE, different MIMO settings, bandwidth, decoding iterations, etc.). Therefore, one should use the power consumption values of the RRU and BBU based on the common base station configurations used in the mobile network. 
A BBU is normally used in a macro base station site, which consists of 3 sectors, to process both uplink and downlink data. Therefore, the variable component of the BBU power consumption represents the increase in BBU power consumption due to the increase in both the uplink and downlink resource utilizations. In contrast, one RRU is used in each sector of a macro base station site (which normally consists of 3 sectors). The increase in the variable power of the RRU mainly corresponds to the increase in power consumption of the power amplifier (PA) for downlink data and signaling transmission.

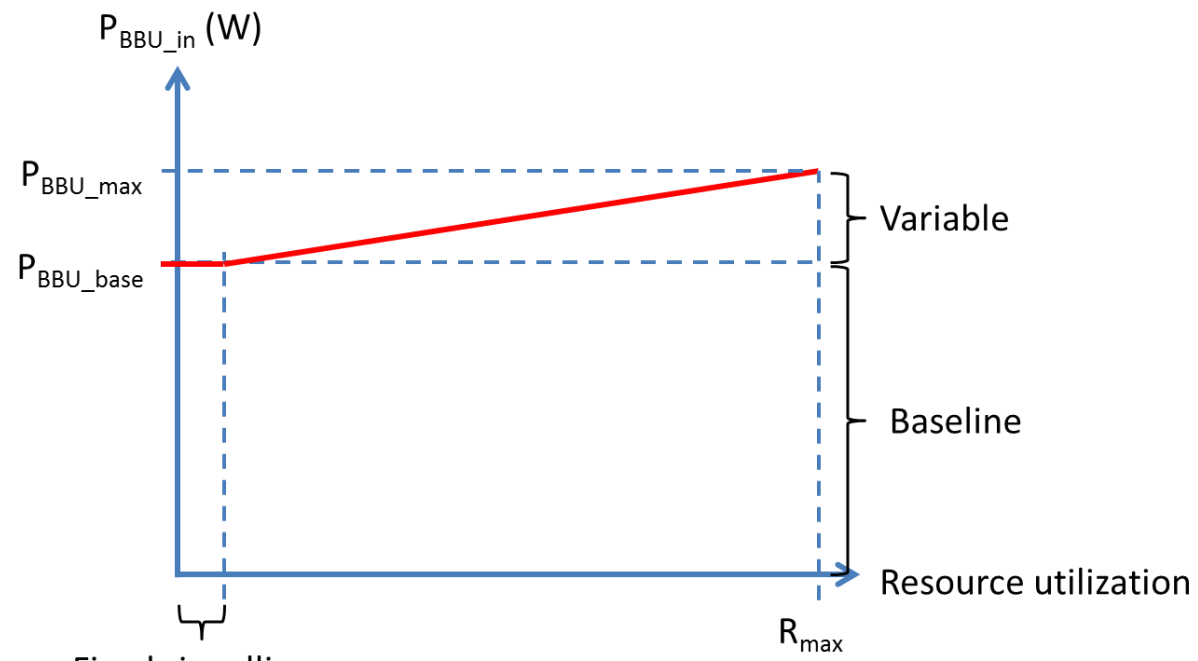

Fixed signalling

Figure 2. Power consumption of the BBU as a function of radio resource utilization.

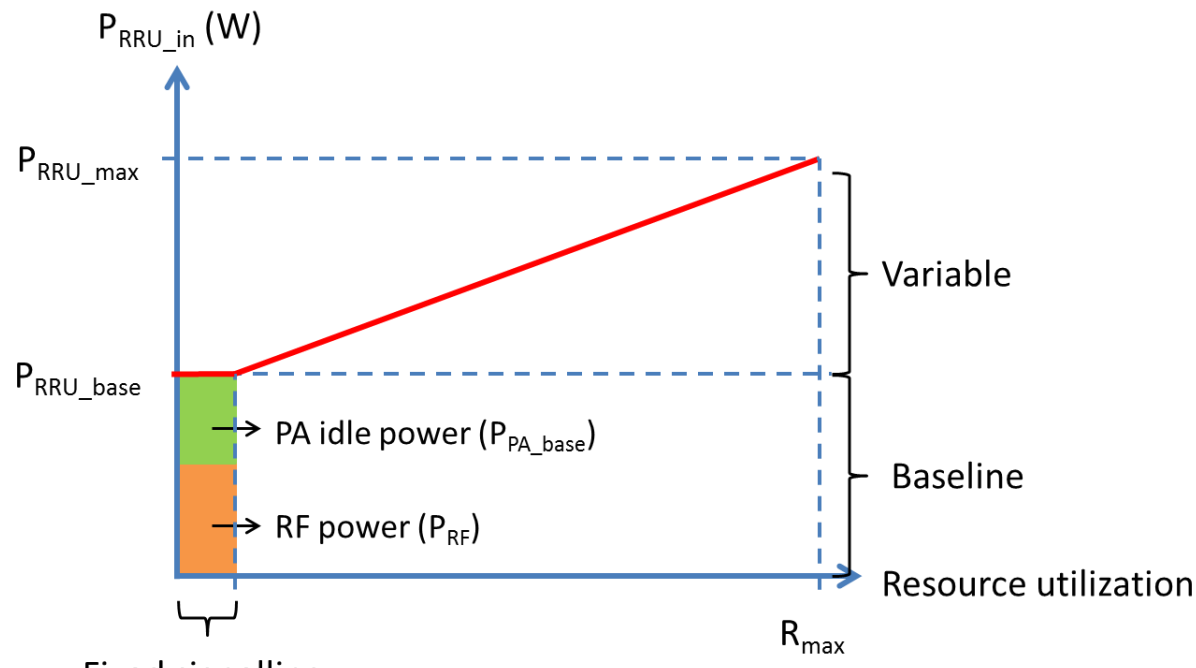

Fixed signalling

Figure 3. Power consumption of the RRU as a function of radio resource utilization. 


\subsection{Power and energy consumption of mobile services}

The total network energy consumption, $E_{\text {service }}$, of a mobile service is given by:

$$
E_{\text {service }}=\int_{0}^{\tau_{s}} P_{S}(t) d t+\int_{0}^{\tau_{d}} P_{d}(t) d t
$$

where $P_{s}(t)$ is the network signaling power consumption of the service at time $t, P_{d}(t)$ is the network data power consumption of the service at time $t$, and $\tau_{s}$ and $\tau_{d}$ are the durations of the signaling and data of the service, respectively. The parameter $\tau_{d}$ is directly dependent on the size of the data of the mobile service and the number of PRBs allocated by the mobile network to the mobile device for the specific mobile service. The size of the data depends on the types of mobile services used. For example, the data size of sending an instant message will be very low compared to a 1-minute voice call as shown in the next section. The number of PRBs allocated by the mobile network to the mobile device is dependent on the network configuration and the load of the cell at the time the mobile service is executing. The parameter $\tau_{d}$ can be either predefined by the mobile operator based on statistics of a specific mobile service or collected via real measurements. Before the mobile device is allocated to any PRBs to transmit data, the mobile device has to setup an RRC connection to enter the connected state from the idle state. After the transmission of the data is completed, the mobile device will enter the idle state. This occurs after a certain period of time called the inactivity time. Therefore, $\tau_{s}$ is expected to be larger than $\tau_{d}$ due to (a) setting up of the RRC connection to request network resources for data transmissions, and (b) tearing down of the connection after timeout of the inactivity timer. Assuming that the RRC connection could be set up effectively between the mobile network and the mobile device with negligible delay, the difference between $\tau_{d}$ and $\tau_{s}$ (i.e., $\tau_{s}-\tau_{d}$ ) is mainly due to the inactivity timer set by the mobile operator. According to China Mobile, the inactivity timer for its nation-wide 4G LTE network is set at 10 seconds. Therefore, we assume that $\tau_{s}$ will be at least 10 seconds longer than $\tau_{d}$. However, the inactivity timer settings for different networks in different countries 
vary so one should use the recommended settings given by local mobile operators. It should also be noted that if a wireless network air interface test tool is used to measure the data and signaling traffic generated by a mobile service, the setting of $\tau_{s}$ in the energy calculation must not be longer than the RRC inactivity timer. This is necessary to avoid capturing signaling overhead traffic that is not related to the mobile service, such as signaling messages generated through PSS, SSS, and PBCH.

\subsubsection{Service signaling power consumption $\left(P_{s}(t)\right)$}

The service signaling power consumption at time $t, P_{s}(t)$ comprises the power consumed at the RRU $\left(P_{s, R R U}(t)\right)$ and the BBU $\left(P_{s, B B U}(t)\right)$ to transmit and process the signaling traffic of the mobile service. $P_{s}(t)$ is given by:

$$
P_{S}(t)=P_{S, R R U}(t)+P_{S, B B U}(t)
$$

For both $P_{s, R R U}(t)$ and $P_{s, B B U}(t)$, we adopt a linear approach by apportioning the power of different base station components (i.e., RRU or BBU) to a mobile service based on how many resource elements (REs) out of the maximum available REs have been utilized by the mobile service over a certain period of time. Therefore, a general equation can be used to determine the signaling power consumption of a mobile service in the $k^{\text {th }}$ component (i.e., RRU or BBU) of the base station:

$$
P_{s, k}(t)=\frac{P_{k}}{R_{\max }} \cdot R_{s}(t)
$$

where $P_{s, k}(t)$ represents the amount of power from component $k\left(P_{k}\right)$ that has been allocated to the service based on how many signaling RE/s $\left(R_{s}(t)\right)$ out of the maximum available RE/s $\left(R_{\max }\right)$ have been used by the service over time. One could use the functional unit of PRBs when performing the calculations ( $1 \mathrm{PRB}=84 \mathrm{REs})$. We use $\mathrm{RE} / \mathrm{s}$ to represent network resource load in this paper because the measurements are obtained in terms of RE/s. Then, we define $\eta_{s, k}$ as the energy (in joules) consumed by one RE in component $k$ : 


$$
\eta_{s, k}=\frac{P_{k}}{R_{\max }} .
$$

By inserting equation (4) into (3) and then (2), equation (2) now becomes:

$$
P_{S}(t)=P_{S, R R U}(t)+P_{S, B B U}(t)=\left(\eta_{s, R R U}+\eta_{s, B B U}\right) R_{S}(t) .
$$

A key challenge in determining $\eta_{S, R R U}$ and $\eta_{S, B B U}$ is to identify the appropriate amount of power to be allocated to each RE in the RRU and the BBU because the way of calculating $P_{k}$ is different for different base station components. Table 1 shows the calculation methods for determining $\eta_{s, R R U}$ and $\eta_{s, B B U}$.

Table 1. Calculation methods for $\eta_{s, R R U}, \eta_{s, B B U}, \eta_{d, R R U}$, and $\eta_{d, B B U}$ for equations (5) and (13). The explanation of the notation and the rationale of the methods are summarized in

\begin{tabular}{|c|c|}
\hline Parameter & Calculation method \\
\hline$\eta_{s, R R U}$ & 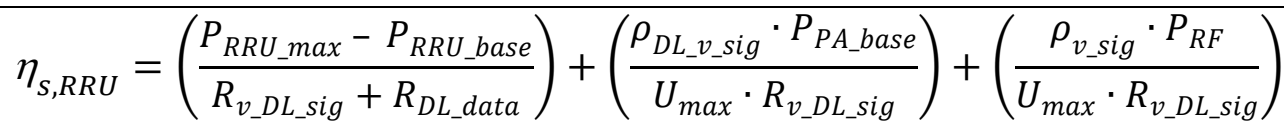 \\
\hline$\eta_{s, B B U}$ & 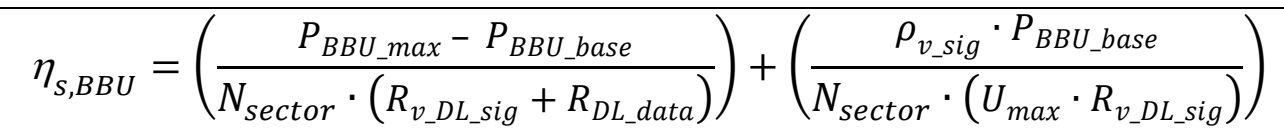 \\
\hline$\eta_{d, R R U}$ & 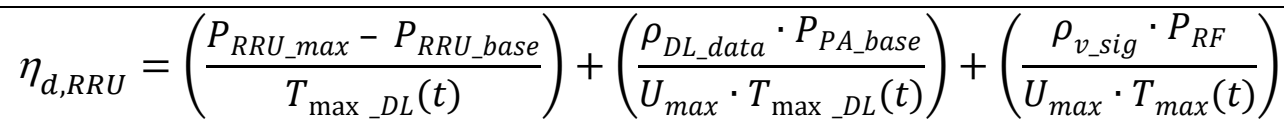 \\
\hline$\eta_{d, B B U}$ & $\eta_{d, B B U}=\left(\frac{P_{B B U \_\max }-P_{B B U \_b a s e}}{N_{\text {sector }} \cdot T_{\max }(t)}\right)+\left(\frac{\rho_{v_{\text {_sig }}} \cdot P_{B B U_{\text {_base }}}}{N_{\text {sector }} \cdot U_{\max } \cdot T_{\max }(t)}\right)$ \\
\hline
\end{tabular}
Section 2.2.3.

\subsubsection{Service data power consumption}

Similar to the approach we use to determine $P_{s}(t), P_{d}(t)$ comprises the power consumed at the RRU $\left(P_{d, R R U}(t)\right)$ and the $\mathrm{BBU}\left(P_{d, B B U}(t)\right)$ to transmit and process the data traffic of the mobile service:

$$
P_{d}(t)=P_{d, R R U}(t)+P_{d, B B U}(t) .
$$

Ideally, $P_{d, R R U}(t)$ and $P_{d, B B U}(t)$ can be determined using equation (7): 


$$
P_{d, k}(t)=\frac{P_{k}}{R_{\max }} \cdot R_{d}(t)
$$

where $P_{d, k}(t)$ represents the amount of power from component $k\left(P_{k}\right)$ that has been allocated to the service based on how many data RE/s $\left(R_{d}(t)\right)$ out of the maximum available RE/s $\left(R_{\max }\right)$ have been used by the service. In order to use equation (7), we require the information of the channel quality indication (CQI) of the connecting mobile device to the base station as the CQI contains information on the modulation format and the coding scheme used by the mobile device when using the mobile service over time. Such information can then be used to determine $R_{d}(t)$ using the CQI table given by [20]. If the fine-grained information on device CQI over time is available, one should use equation (7) to perform the calculation. However, the fine-grained device CQI information cannot currently be collected from China Mobile's network monitoring systems. Instead, the measurements of the service data rate (in bits per second), the base station's average throughput and PRB utilization (over every 15 minute time interval) are available from the network monitoring systems of China Mobile. Therefore, we modify equation (7) to calculate the average data energy consumption of a mobile service using the available information above. It should be noted that this service data energy could vary when different users are using the same service over the same time in the same cell site due to different CQIs as mentioned earlier.

We substitute $R_{d}(t)$ in equation (7) with $T_{d}(t)$, which is the data rate (in bits per second) of the mobile service. The relationship between $R_{d}(t)$ and $T_{d}(t)$ is shown in the following equation:

$$
T_{d}(t)=k_{d}(t) \cdot R_{d}(t)
$$

where $k_{d}(t)$ is the average amount of data that can be carried by each RE allocated to the mobile device for the specific service at time $t$ and $k_{d}(t)$ has a unit of bits/RE. $T_{d}(t)$ can be either predefined by the mobile operator based on statistics of a specific mobile service or 
collected via real measurements. In this paper, we use real measurements to determine $T_{d}(t)$ as will be discussed in Section 3.

In order to substitute the number of REs per second $(R)$ with throughput or data rate $(T)$ in bits per second for equation (3), we need to determine $T_{\max }$, which is the maximum throughput (bits per second) of the base station. Similar to equation (8), the relationship between $R_{\max }$ and $T_{\max }$ can be represented by the equation:

$$
T_{\max }(t)=k_{m}(t) \cdot R_{\max }
$$

where $k_{m}(t)$ is the average number of bits per RE per second offered by the base station at time $t . T_{\max }(t)$ can be obtained by dividing the base station actual throughput and PRB utilization measurements at time $t$. Substituting equations (8) and (9) into equation (7) to determine $P_{d, k}(t)$, equation (7) now becomes:

$$
\left.P_{d, k}(t)=\frac{P_{k}}{\left(T_{\max }(t) / k_{m}(t)\right.}\right) \cdot\left(T_{d}(t) / k_{d}(t)\right)
$$

It should be noted that $k_{m}$ is the average bits/RE for the base station for all $N$ connecting mobile devices, while $k_{d}$ is the bits/RE for one particular mobile device using the mobile service where $k_{d}$ is dependent on the CQI of the mobile device. Since $k_{d}(t)$ is unavailable in the current network measurements from China Mobile, we simply assume that $k_{d}(t)=k_{m}(t)$. This assumption works well for cell sites with small coverage (e.g., urban areas) because the variation in the value of $k$ of each user will not be significant compared to big cells (e.g., rural areas). Since we are using base station measurements from Beijing urban cell sites, which have a coverage of less than $1 \mathrm{~km}^{2}$ according to China Mobile, we assume that $k_{d}(t) \approx k_{m}(t)$ and hence equation (10) now becomes: 


$$
P_{d, k}(t)=\frac{P_{k}}{T_{\max }(t)} \cdot T_{d}(t)
$$

It should be noted that $T_{d}(t)$ equals to the service downlink data rate when calculating the downlink data power consumption and $T_{d}(t)$ equals service uplink data rate when calculating the uplink data power consumption. Hence, we define $\eta_{d, k}$ as the power (in watts) consumed by one bit per second of data rate in component $k$ at time $t$ :

$$
\eta_{d, k}=\frac{P_{k}}{T_{\max }(t)}
$$

By inserting equation (12) into (11) and then (6), equation (6) changes to:

$$
P_{d}(t)=P_{d, R R U}(t)+P_{d, B B U}(t)=\left(\eta_{d, R R U}+\eta_{d, B B U}\right) T_{d}(t)
$$

Similar to $\eta_{s, R R U}$ and $\eta_{s, B B U}$, a key challenge in determining $\eta_{d, R R U}$ and $\eta_{d, B B U}$ is to identify the appropriate power components to be allocated to the data traffic of the mobile service in the RRU and the BBU because the way of calculating $P_{k}$ is different for different components. Table 1 shows the calculation method to determine $\eta_{d, R R U}$ and $\eta_{d, B B U}$.

\subsubsection{Calculation methods of $\eta_{s, R R U}, \eta_{s, B B U}, \eta_{d, R R U}$, and $\eta_{d, B B U}$}

The descriptions of the notation and the explanations of the calculation methods in Table 1 are as follows:

- $\quad \eta_{s, R R U}$ (energy (in joules) consumed by one RE in the RRU): The first term of the method calculates the variable power of the RRU consumed by each signaling RE by dividing the variable power component of the RRU $\left(P_{R R U_{-} \max }-P_{R R U_{-} b a s e}\right)$ by all available REs for downstream transmissions $\left(R_{v_{-} D L_{-} s i g}+R_{D L_{-} d a t a}\right)$, where $R_{v_{-} D L_{-} s i g}$ is the total REs available for variable signaling (i.e., physical downlink control channel) per second and $R_{D L \_d a t a}$ is the total REs available for downlink data per second (i.e., physical downlink shared channel). The second term calculates the baseline power of the PA consumed by each signaling RE by dividing the signaling power component of the PA $\left(\rho_{D L_{-} v_{-} \text {sig }} \cdot P_{P A \_b a s e}\right)$ 
by the available signaling REs $\left(U_{\max } \cdot R_{v_{-} D L_{-} s i g}\right) . \rho_{D L_{-} v_{-} s i g}$ is the ratio of the total REs available for variable downlink signaling to the total REs for downlink including data, fixed and variable signaling and $U_{\max }$ is the maximum utilization threshold of the base station set by a policy decision of the mobile operator to ensure that the base station is not constantly fully loaded. The third term calculates the amount of RF power consumed by each signaling RE by dividing the signaling power component of the RF $\left(\rho_{v_{-} s i g} \cdot P_{R F}\right)$ by $\left(U_{\text {max }} \cdot R_{v_{-} D L_{-} s i g}\right)$, where $\rho_{v_{-} s i g}$ is the ratio of the total REs available for downlink variable signaling to the total REs for the uplink and downlink including data, fixed and variable signaling.

- $\quad \eta_{s, B B U}$ (energy (in joules) consumed by one RE in the BBU): The first term calculates the variable power of the BBU per sector consumed by each signaling RE by dividing $\left(\left(P_{B B U_{-} \text {max }}-P_{B B U_{-} \text {base }}\right) / N_{\text {sector }}\right)$ by all available REs $\left(R_{v_{-} D L_{-} s i g}+R_{D L_{-} \text {data }}\right)$. It should be noted that because a BBU is typically used in a macro base station site that consists of 3 sectors to process both uplink and downlink data, $N_{\text {sector }}$ is included in the equation to divide the variable power of a BBU on a per sector basis. The second term calculates the baseline power of the BBU consumed by each signaling RE by dividing the baseline signaling power component of the $\mathrm{BBU}\left(\rho_{v_{-} s i g} \cdot P_{B B U_{-} b a s e}\right)$ by all signaling REs $\left(U_{\max } \cdot R_{v_{-} D L_{-} s i g}\right)$.

- $\quad \eta_{d, R R U}$ (energy (in joules) consumed by one bit of data in the RRU): The first term calculates the variable power of the RRU consumed by one bit of data traffic by dividing the variable power component of the RRU $\left(P_{R R U_{-} \text {max }}-P_{R R U_{-} \text {base }}\right)$ by the maximum throughput of the base station $\left(T_{\text {max } D L}(t)\right)$. It should be noted that only downlink data are taken into account for RRU because the variable power of the RRU (i.e., PA power) is mainly for downlink transmissions. The second term calculates the amount of baseline power of the PA consumed by one bit of data traffic by dividing the data power 
component of the PA $\left(\rho_{D L_{-} d a t a} \cdot P_{P A \_b a s e}\right)$ with $\left(U_{\max } \cdot T_{\text {max } \_D L}(t)\right)$ where $U_{\max }$ is the maximum utilization threshold of the base station set by a policy decision of the mobile operator. It should be noted that the first two terms are mainly for downstream transmissions. Therefore, if one is calculating the uplink data energy consumption of the service, the first two terms can be omitted.

- $\quad \eta_{d, B B U}$ (energy (in joules) consumed by one bit of data in the RRU): The first term calculates the variable power of the BBU per sector consumed by one bit of data traffic by dividing the variable power component of the BBU $\left(\left(P_{B B U_{-} \text {max }}-P_{B B U_{-} \text {base }}\right) / N_{\text {sector }}\right)$ by the maximum throughput of the base station $\left(T_{\max }(t)\right)$, where $T_{\max }(t)$ is the maximum throughput (for both uplink and downlink) of the base station at time $t$. The second term calculates the baseline power of the BBU consumed by one bit of data traffic by dividing the baseline power component of the BBU allocated for all data $\left(\left(\rho_{\text {data }} \cdot P_{B B U \_b a s e}\right) / N_{\text {sector }}\right)$ by the maximum throughput of the base station $\left(U_{\max } \cdot T_{\max }(t)\right)$.

In the calculation methods of $\eta_{S, R R U}$ and $\eta_{d, R R U}$, the parameters $P_{P A_{-} b a s e}$ and $P_{R F}$ can be determined from vendor specifications. Alternatively, $P_{P A_{-} b a s e}$ and $P_{R F}$ can be determined using equations (14) and (15), respectively:

$$
\begin{gathered}
P_{P A_{\_} \text {base }}=N_{T X} \cdot \frac{P_{P A_{\_} \text {out }}}{\eta_{P A}} \cdot R_{P A_{\_} \text {base } / \text { max }}, \\
P_{R F}=P_{R R U_{\text {base }}}-P_{P A \_b a s e},
\end{gathered}
$$

where $N_{T X}$ is the number of antennas. If a $2 \times 2 \mathrm{MIMO}$ system is used, $N_{T X}$ is 2 . $P_{\text {PA_out }}$ is the maximum output power of the PA. For example, the maximum power consumption of the PA for an LTE macro base station is normally $40 \mathrm{~W}$ per transmitter. $\eta_{P A}$ is the in-efficiency factor for PA (e.g., 0.35 [23]) and $P_{P A \_b a s e / m a x}$ is the ratio of the baseline power to the maximum power of the PA (e.g., 1/6 based on real measurements). 


\section{Comparing network energy consumption of OTT IM services and conventional}

\section{mobile services}

In this section, we use our energy models to compare the network energy consumption of WeChat services and conventional mobile services. As discussed in the previous sections, there has been a recent change in user behavior towards the preference for using OTT IM applications over conventional mobile services to send text, picture and voice messages. As observed in China Mobile's networks, this change in user behavior will not only decrease the usage of SMS, MMS and voice calls, it will also cause a large amount of network energy consumption. Therefore, operators are interested in exploring whether the use of OTT IM applications to send text, picture and voice messages will be more or less energy-efficient than sending SMS, MMS and voice calls.

\subsection{Input parameters to the energy models}

The power consumption values of the base stations $\left(P_{B B U_{-} \max }, P_{B B U_{-} b a s e}, P_{R R U_{-} \max }\right.$, $P_{R R U \_b a s e}, P_{R F}$, and $\left.P_{P A_{-} b a s e}\right)$ are shown in Table 2 . These power consumption values are based on real measurements conducted by the China Mobile Research Institute. The downlink and uplink maximum throughput values $\left(T_{\max \_D L}, T_{\max \_U L}\right)$ shown in Table 2 are based on average values collected from 3 cell sites in Beijing's urban areas over 7 days in October 2014. The network resources parameters, i.e., $\rho_{\text {data }}, \rho_{v_{-} s i g}, \rho_{D L_{-} d a t a}, \rho_{D L_{-} v_{-} s i g}, R_{v_{-} D L_{-} s i g}$, and $R_{D L_{-} \text {data }}$ shown in Table 2 are calculated based on 3GPP standards. As mentioned in Section 2, $\rho_{\text {data }}, \rho_{v_{-}}$sig, $\rho_{D L_{-} \text {data }}, R_{v_{-} D L_{-} \text {sig }}$, and $R_{D L_{-} \text {data }}$ are dependent on the base station configuration, such as the bandwidth, the TD-LTE sub-frame configuration, the number of antennas (which varies with the number of CRS per sub-frame), the PHICH values, and the number of physical downlink control channel symbols per sub-frame. 
Table 2. Measurement parameters of TD-LTE mobile access networks.

\begin{tabular}{|c|c|c|c|}
\hline Parameters & Values & Parameters & Values \\
\hline Power consumption & & Network resources & \\
\hline$P_{B B U \_\max }$ & $107.67 \mathrm{~W}$ & $\rho_{\text {data }}$ & 0.74 \\
\hline$P_{B B U \_b a s e}$ & $103.50 \mathrm{~W}$ & $\rho_{v_{-} s i g}$ & 0.16 \\
\hline$P_{R R U \_\max }$ & $202.50 \mathrm{~W}$ & $\rho_{D L \_ \text {data }}$ & 0.50 \\
\hline$P_{R R U \_b a s e}$ & $84.30 \mathrm{~W}$ & $\rho_{D L_{-} v_{-} s i g}$ & 0.2127 \\
\hline$P_{R F}$ & $46.20 \mathrm{~W}$ & $R_{v_{-} D L \_s i g}$ & $\begin{array}{l}2,307,200 \\
\mathrm{RE} / \mathrm{s}\end{array}$ \\
\hline$P_{P A \_b a s e}$ & $38.10 \mathrm{~W}$ & $R_{D L \_d a t a}$ & $\begin{array}{l}9,466,400 \\
\mathrm{RE} / \mathrm{s}\end{array}$ \\
\hline \multicolumn{4}{|c|}{ Average cell site throughput (per sector) } \\
\hline$T_{\text {max_DL }}$ & $120 \mathrm{Mbps}$ & & \\
\hline$T_{\max \_U L}$ & $20 \mathrm{Mbps}$ & & \\
\hline
\end{tabular}

Measurement parameters such as $T_{d}$ and $\tau_{d}$ for conventional mobile services are determined through real measurements. We capture the information listed in Table 3 using the Pilot Pioneer Wireless Network Air Interface Test Tool from Ding Li Communications Corp., Ltd [24]. This test tool is capable of analyzing signaling and data packet information transmitted via the air interface. A smartphone was first connected to a computer and configured to connect to $4 \mathrm{G}$ networks only. Then, test scripts and test information were loaded into the smartphone to automate the testing procedure for different mobile services listed under the Description of Table 3. The same testing procedure was repeated six times and we found small variations in the parameters $T_{d}$ and $\tau_{d}$ shown in Table 3. From our measurements, the standard deviation of $T_{d}$ for SMS is +/- $18 \%$ and the $\tau_{d}$ of SMS is constantly 1 second. For MMS, the standard deviations of $T_{d}$ and $\tau_{d}$ for MMS are $+/-9 \%$ and $+/-20 \%$, respectively. 
For WeChat services, the average data size of the text message, $B_{d_{-} \text {text }}$ defined as $\left(B_{d_{-} \text {text }}=T_{d} \times \tau_{d}\right)$ is 732 bytes. This includes the 298 bytes generated by the hint message and 434 bytes generated by the data when considering a text message which consists of 58 Chinese characters. The average data duration for text messages is estimated based on the interval time between the hint message and the entire text message. It should be noted that our estimation is conservative because we assume that the user will start typing (i.e., the hint message will be sent) and send the message within a few seconds. If the interval between hint message and the entire message is large, more network energy will be consumed. The following equation can be used to estimate the data size of the test message comprising different numbers of characters [25]:

$$
B_{d_{-} t e x t}=B_{\text {hint }}+B_{\text {text }}
$$

where $B_{\text {hint }}$ corresponds to the data packets generated by the hint messages, which is estimated to be 298 bytes and $B_{\text {text }}$ corresponds to the data packets generated by the total number of characters in the text message. For English text messages, $B_{\text {text }}$ equals 386 bytes for 10 English characters, 402 bytes for 11 to 26 English characters and 418 bytes for 27 to 42 English characters, with an increase of 16 bytes for every additional 16 English characters. For Chinese text messages, $B_{\text {text }}$ equals 386 bytes for 1 to 3 Chinese characters, 402 bytes for 4 to 7 Chinese characters and 418 bytes for 8 to 11 Chinese characters, with an increase of 16 bytes for every additional 4 Chinese characters.

For sending a picture via WeChat, we found that WeChat compressed all pictures being sent and received. Based on our measurements, most of the pictures received have been compressed to a size of around 70 kbytes. However, in the case of sending a picture via MMS, the picture will be uncompressed. For voice messages, the maximum time length of a voice message allowed in WeChat is 60 seconds. Each second of the voice message will incur 0.9 kbytes of data followed by a 66-byte end flag at the end of the transmission. The average data 
rate for a voice message is estimated based on our real measurements of cloud upload and download of multimedia files.

Without loss of generality, we fix the size of the text message, voice message and picture for our assessment in this paper. One could use the energy models and network measurement parameters listed in Table 2 together with real measurements or equation (16) for different sizes of messages to conduct similar assessments.

Table 3. Measurement parameters of WeChat and conventional mobile services.

\begin{tabular}{|c|c|c|c|}
\hline Services & Description & $\begin{array}{l}\text { Average data } \\
\text { rate }\left(T_{d}\right) \text { in } \\
\text { kbits/s }\end{array}$ & $\begin{array}{c}\text { Average data } \\
\text { duration }\left(\tau_{d}\right) \text { in } \\
\text { seconds }\end{array}$ \\
\hline \multicolumn{4}{|l|}{$\begin{array}{l}\text { Conventional mobile } \\
\text { services }\end{array}$} \\
\hline Send SMS & 58 Chinese characters & 2 & 1 \\
\hline Receive SMS & 58 Chinese characters & 2 & 1 \\
\hline Send MMS & $\begin{array}{l}\text { Send a picture with size of } \\
112 \text { kbytes }\end{array}$ & 47.16 & 19 \\
\hline Receive MMS & $\begin{array}{l}\text { Receive a picture with size of } \\
112 \text { kbytes }\end{array}$ & 47.16 & 19 \\
\hline Voice call & $\begin{array}{l}\text { Voice call duration of } 60 \\
\text { seconds }\end{array}$ & 13.3 & 60 \\
\hline \multicolumn{4}{|l|}{ OTT IM services } \\
\hline Send a text message & 58 Chinese characters & 1.1712 & 5 \\
\hline Receive a text message & 58 Chinese characters & 1.1712 & 5 \\
\hline Send a picture & Size of picture $=70$ kbytes & 186.67 & 3 \\
\hline Receive a picture & Size of picture $=70$ kbytes & 186.67 & 3 \\
\hline Send a voice message & $\begin{array}{l}\text { Contain } 30 \text { seconds of voice } \\
\text { information }\end{array}$ & 240 & 5 \\
\hline $\begin{array}{l}\text { Receive a voice } \\
\text { message }\end{array}$ & $\begin{array}{l}\text { Contain } 30 \text { seconds of voice } \\
\text { information }\end{array}$ & 240 & 5 \\
\hline Heartbeat & $\begin{array}{l}\text { To maintain 'always-on' with } \\
\text { server and check for updates }\end{array}$ & 0.656 & 1 \\
\hline
\end{tabular}


As mentioned in the previous sections, unlike conventional mobile services, OTT IM applications require frequent $\mathrm{RRC}$ connection reestablishments to keep the mobile users in touch with the servers, which is known as a 'heartbeat' or 'keep-alive'. These periodic messages keep the application state up-to-date and inform the user of the time in case of any event $[14,16]$. Although the data size of these messages is quite small, the frequency of heartbeat messages will generate a large amount of network signaling energy. Therefore, it is important for us to understand the energy consumption of heartbeat messages in OTT IM applications. In Wechat, the data size of a heartbeat packet is 82 bytes [25]. We assume that Wechat's heartbeat interval during an idle period is 5 minutes [26].

For the average signaling traffic $\left(R_{s}\right)$ of mobile services, through real measurements of different types of mobile services, we found that $R_{s}$ varies between $120 \mathrm{RE} / \mathrm{s}$ to $190 \mathrm{RE} / \mathrm{s}$ with an average of $144 \mathrm{RE} / \mathrm{s}$. Therefore, we assume that $R_{s}$ equals $144 \mathrm{RE} / \mathrm{s}$ in our calculations. Furthermore, as mentioned in Section 2.2, we assume $\tau_{s}$ is 10 seconds longer than $\tau_{d}$ because according to China Mobile Network Operations, the network RRC inactivity time for China Mobile's LTE network is set to 10 seconds.

\subsection{Network energy consumption of mobile services}

Using the energy models presented in Section 2 with the parameters presented in Tables 2 and 3, the network energy consumption of each service is shown in Figure 4. The network energy consumption of each service includes the signaling energy and data energy to transmit and receive one message excluding the heartbeat. As shown in Figure 4, sending a text message using WeChat consumes 0.0148 joules of network data energy and 0.1110 joules of network signaling energy. In contrast, an SMS consumes 0.0051 joules of network data energy and 0.0814 joules of network signaling energy. As a result, WeChat consumes more network energy on the text messaging service compared to conventional SMS. This is due to more energy being consumed by the hint messages, which are used to notify the recipient 
when the sender has started typing a message. By sending a hint message a few seconds before the entire message, it increases the time in the RRC connected state, which will incur additional network signaling overheads. In general, network signaling energy accounts for the majority of energy consumed for text messages (88\% and $94 \%$ of the total energy consumption for WeChat and SMS, respectively).

For sending a picture, WeChat consumes 1.4175 joules of network data energy and 0.0962 joules of network signaling energy. In contrast, an MMS consumes 2.2679 joules of network data energy and 0.2145 joules of network signaling energy. As a result, WeChat consumes less network energy to send and receive a picture compared to conventional MMS. This is due to the compression mechanism used in WeChat for pictures as explained in Section 3.1. For a voice message and a conventional call, we compare the energy consumption of 2 WeChat voice messages (30 seconds each) to 1 conventional call with a duration of 60 seconds. Figure 4 shows that WeChat consumes 0.6075 joules of network data energy and 0.0814 joules of network signaling energy. In contrast, a conventional voice call consumes 2.0199 joules of network data energy and 0.2589 joules of network signaling energy. As a result, WeChat consumes less network energy on a voice messaging service compared to a conventional voice call. This is because WeChat records the voice from the user and then encodes this into multiple packets before transmitting the data packets in a burst. In contrast, a conventional call keeps the connection alive throughout the entire call. 


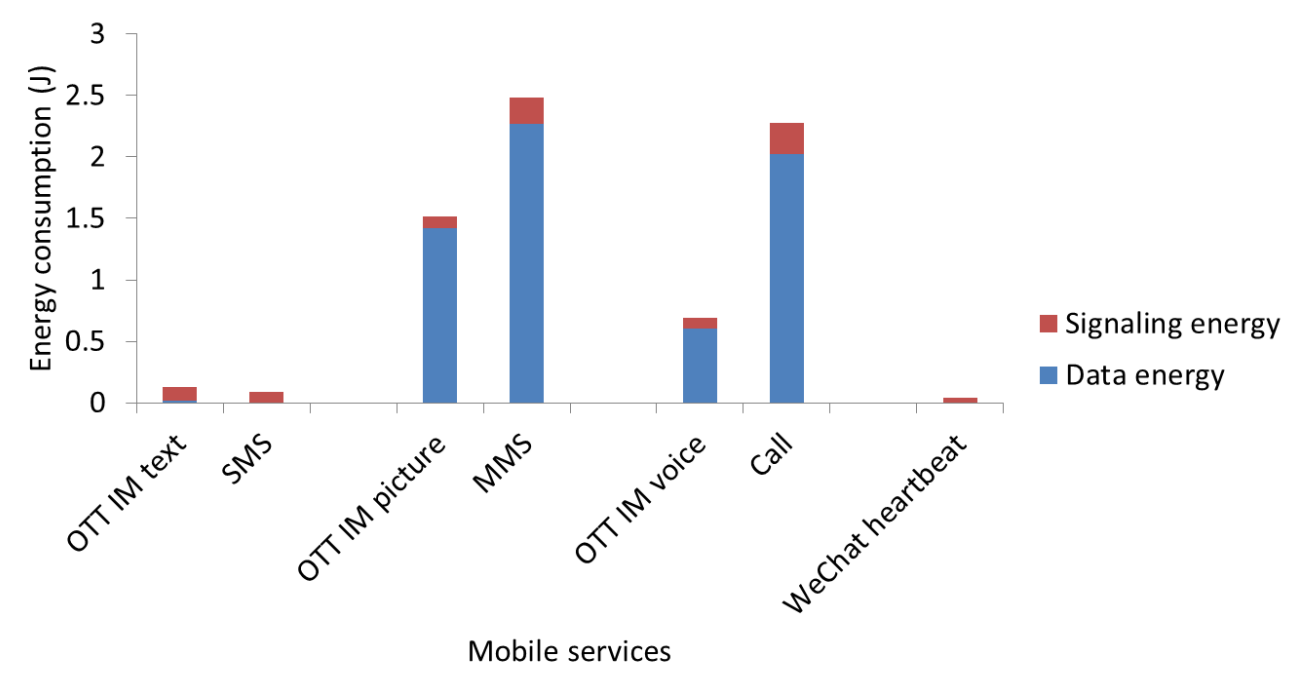

Figure 4. Network energy consumption of WeChat services and conventional mobile services.

\subsection{Network energy consumption based on different user behaviors}

In real world situations, OTT IM users use a combination of text, picture, and voice services. In order to assess whether WeChat or conventional mobile services will be more energy-efficient for a specific type of user, we have to factor in different user behaviors and the daily usage of a user. For example, if a user prefers to send text messages rather than voice messages, then Wechat will be less energy-efficient because text messages consume more energy than SMS. In contrast, using Wechat to send voice messages will be more energy-efficient than a voice call. Therefore, to estimate the network energy consumption of mobile services based on user behavior, we define 11 scenarios of user behaviors with different ratios of text message versus picture versus voice message, as shown in Table 4. The base scenario (Scenario 6) with a text versus picture versus voice ratio of 16:1:5 is extrapolated from the China Mobile Hubei network operations report 2013 [27]. It should be noted that according to this report, the usage of text and voice messaging services is significantly higher than sending/receiving pictures. Therefore, the number of picture 
messages is assumed to be fixed. Scenarios 1 to 5 demonstrate user behaviors that favor voice messaging services. In contrast, Scenarios 7 to 11 demonstrate user behaviors that favor text messaging services. The throughput percentages of these three services for the 11 user behavior scenarios are listed in Table 4.

Using the energy models presented in Section 2 with the parameters presented in Tables 2 and 3, Figure 5 shows the energy ratios of WeChat to conventional mobile services for 11 scenarios of user behaviors. The data show that the energy ratio can be up to a factor of 3 (which means that WeChat consumes 3 times more network energy than conventional mobile services) for daily data volumes of less than 400 kbytes. WeChat consumes less network energy than conventional services in the red region shown in Figure 5. Base scenario users and heavy voice message users with a high daily data volume are within this region. Beyond this region, the network energy consumed by WeChat is much higher than for conventional services by light users and heavy text messaging users.

Table 4. Definition of Different User Behaviors.

\begin{tabular}{|c|c|c|c|c|}
\hline \multirow{2}{*}{ User behavior } & \multirow{2}{*}{$\begin{array}{l}\text { Ratio of text vs. } \\
\text { picture vs. voice } \\
\text { (number of messages) }\end{array}$} & \multicolumn{3}{|c|}{ Percentage of daily data volume } \\
\hline & & Text & Picture & Voice \\
\hline Scenario 1 (Heavy voice) & $2: 1: 200$ & $0.0 \%$ & $1.2 \%$ & $98.8 \%$ \\
\hline Scenario 2 & $4: 1: 150$ & $0.1 \%$ & $1.5 \%$ & $98.4 \%$ \\
\hline Scenario 3 & $6: 1: 100$ & $0.1 \%$ & $2.3 \%$ & $97.6 \%$ \\
\hline Scenario 4 & $8: 1: 50$ & $0.4 \%$ & $4.4 \%$ & $95.2 \%$ \\
\hline Scenario 5 & $10: 1: 25$ & $0.9 \%$ & $8.5 \%$ & $90.7 \%$ \\
\hline Scenario 6 (Base) & $16: 1: 5$ & $5.1 \%$ & $30.2 \%$ & $64.7 \%$ \\
\hline Scenario 7 & $25: 1: 4$ & $8.8 \%$ & $33.6 \%$ & $57.6 \%$ \\
\hline Scenario 8 & $50: 1: 3$ & $18.6 \%$ & $35.6 \%$ & $45.8 \%$ \\
\hline Scenario 9 & $100: 1: 2$ & $36.0 \%$ & $34.4 \%$ & $29.5 \%$ \\
\hline Scenario 10 & $150: 1: 1.5$ & $48.8 \%$ & $31.1 \%$ & $20.0 \%$ \\
\hline Scenario 11 (Heavy text) & 200:1:1 & $59.4 \%$ & $28.4 \%$ & $12.2 \%$ \\
\hline
\end{tabular}


We further break down the service energy consumption into two components: signaling energy (due to the LTE network) and data energy. To compare the signaling energy and the data energy under different user behavior scenarios and different daily data volumes, we define a metric called Signaling to Data Energy Ratio (SDER). The SDER is defined as the ratio of daily network signaling energy consumption to the daily data energy consumption. Figure 6 and 7 show the SDER of conventional services and WeChat, respectively.

Figure 6 shows that the SDER of conventional mobile services is directly related to the ratio of text : picture : voice messages and that it does not depend on the daily data volume. If the user sends more text messages than picture and voice messages (for a ratio above 50:1:3 according to Figure 6), the network signaling energy consumption will be more than $50 \%$ of the total network energy consumption. This is mainly due to the nature of small packet data of text messaging services. For a mobile device to request a connection to the base station to send the text message, a large amount of network signaling traffic is generated, which explains the phenomenon of a high SDER for heavy text users as shown in Figure 6. In contrast, for heavy voice users, because the data sizes of the voice call are large, the signaling overheads are not dominant. Therefore, we observe a low SDER (lower than one) for heavy voice users.

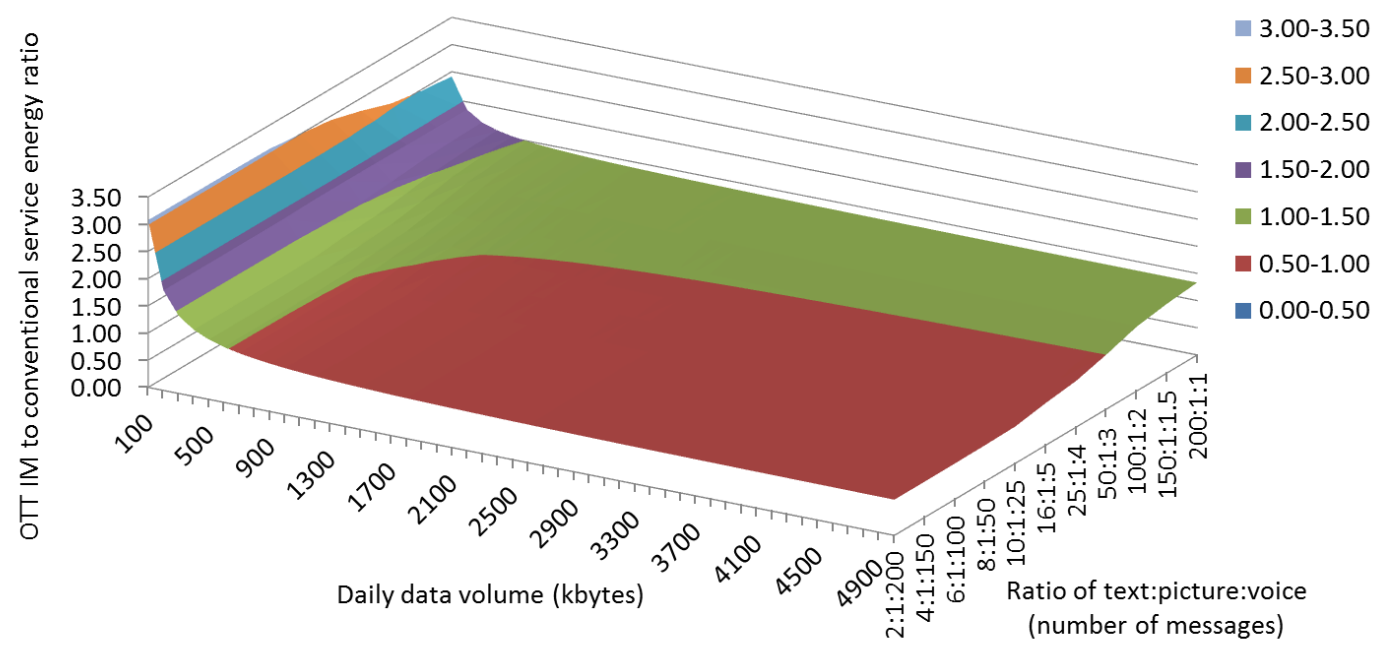


Figure 5. Energy ratio of OTT IM services (i.e., WeChat) to conventional mobile services for different user behaviors and daily data volumes.

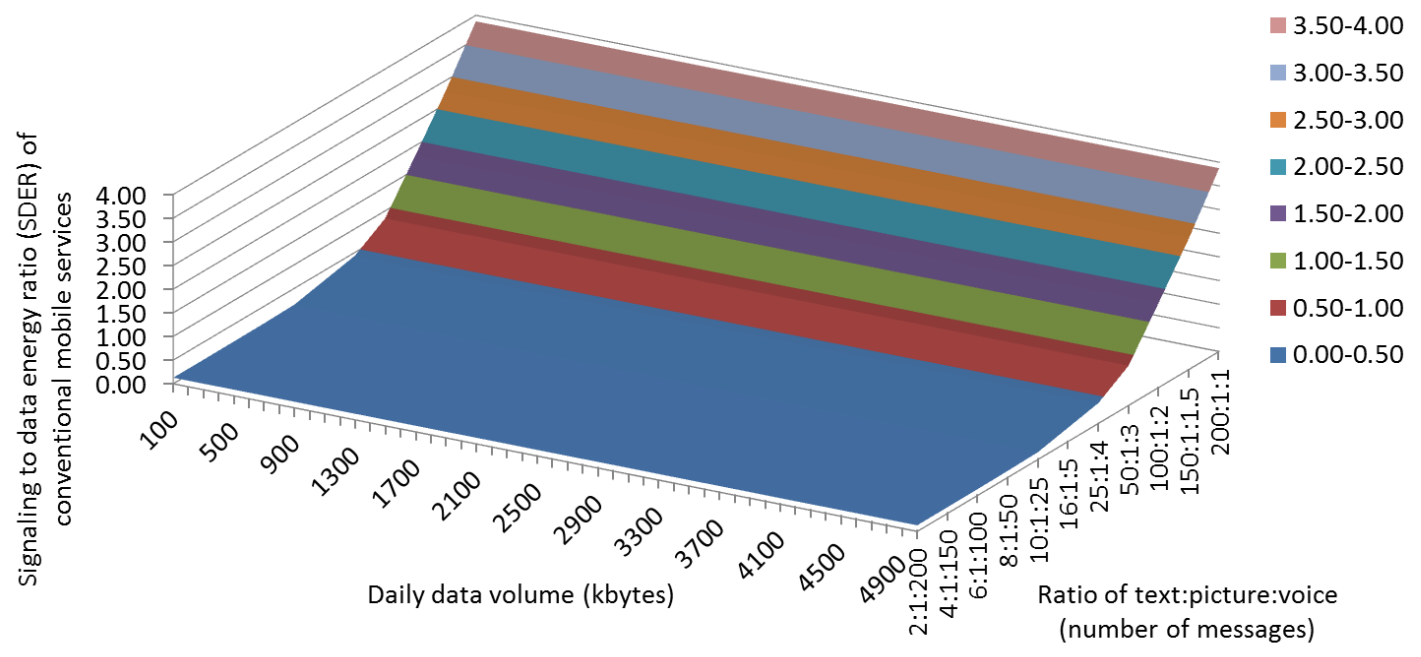

Figure 6. The signaling to data energy ratio of conventional services for different user behaviors and daily data volumes.

In contrast to conventional services, Figure 7 shows that the SDER of OTT IM services for WeChat. For daily data volumes higher than 400 kbytes, the results are similar to conventional mobile services: (1) a high SDER for heavy text users due to network signaling energy overheads for transmissions of small data packets and (2) a low SDER for heavy voice users due to the domination of network data energy. For a daily data volume lower than 400 kbytes, we observe that there is a rise in SDER as the daily data volume decreases. This is due to the fact that the network signaling energy overheads for heartbeats dominate the overall OTT IM service energy consumption. Moreover, when investigating the combined impact of heartbeat and small packet transmission (e.g., a high ratio of text : picture : voice and low daily data volume in Figure 7), the ratio increases sharply, and most network resources are consumed by network signaling in that region. 


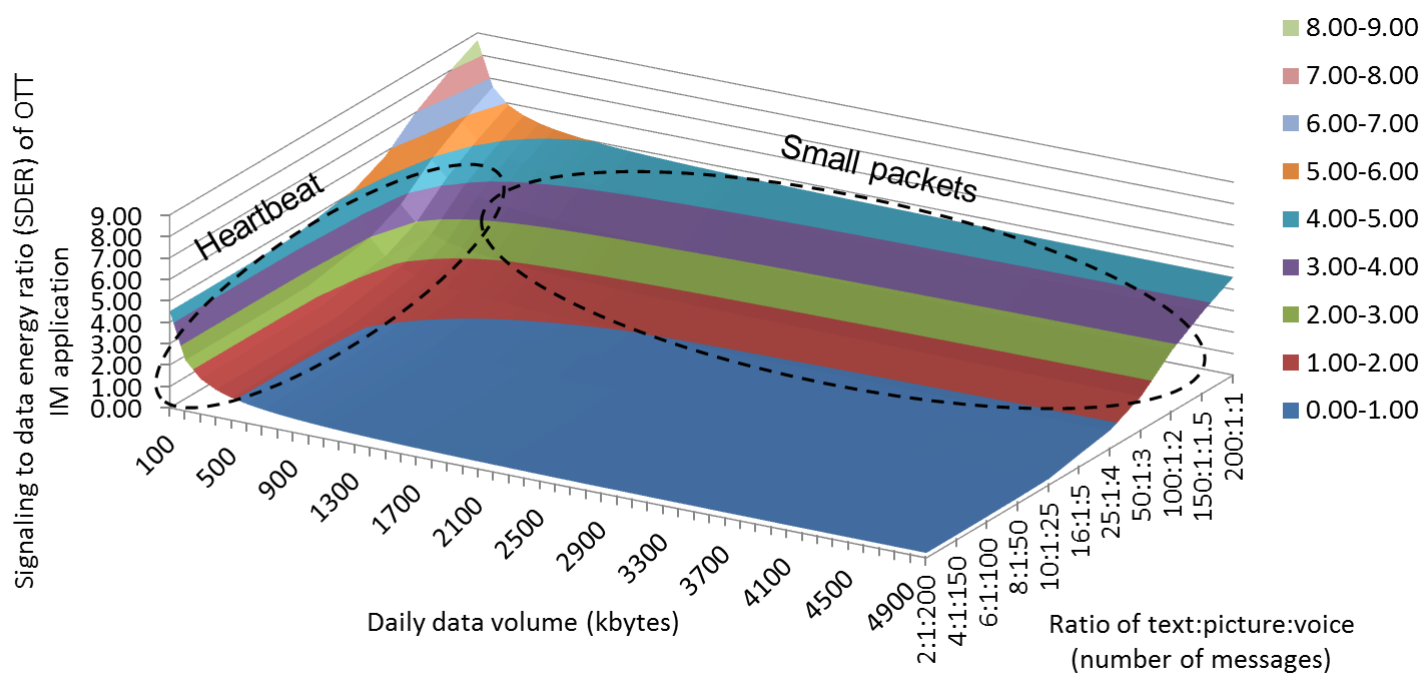

Figure 7. The signaling to data energy ratio of WeChat for different user behaviors and daily data volumes.

\section{Discussion and future work}

In this section, we discuss how our results could assist mobile operators to better understand the behavior of OTT IM services' users so that effective solutions could be developed to reduce the corresponding network energy consumption. Using our energy models, the behavior of OTT IM users can be categorized into four groups based on data transmission volume and service type: (1) heavy big data users, (2) light big data users, (3) heavy small data users, and (4) light small data users, as shown in Figure 8.

Although the aim of the paper is to evaluate the network energy consumption of basic OTT IM functions similar to the conventional mobile services, there are interesting features such as video calls and WeChat "Moments" (which enables users to share and to gain access to their WeChat friends' information, and to create an intimate and private communicating circle within the users' choice of friends), which cannot be provided by conventional mobile services. Therefore, using the energy models proposed in this paper together with real service measurements, the energy consumption evaluations of these features will be an interesting direction for future work. 


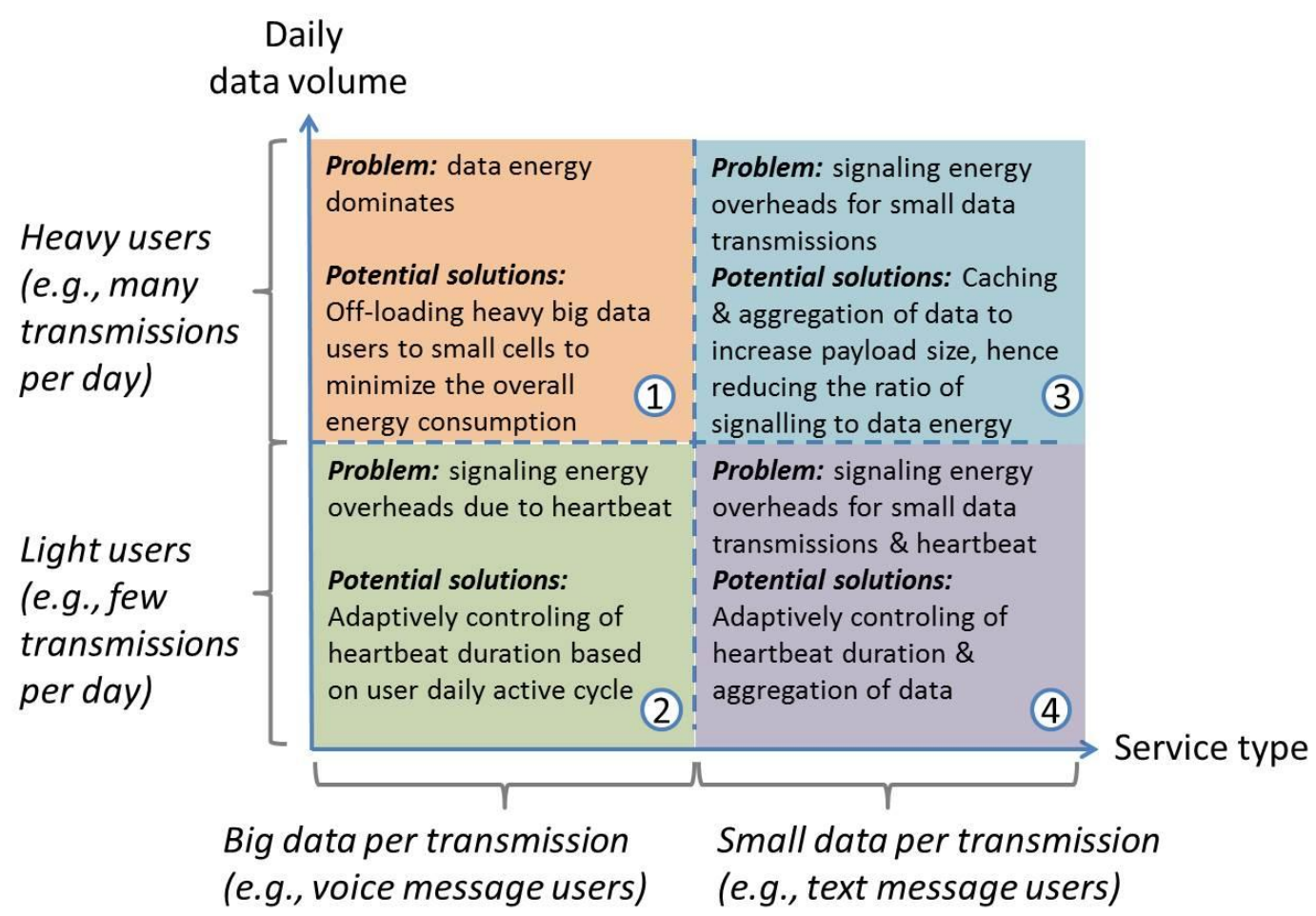

Figure 8. Four-quadrant model to group different OTT IM user behaviors.

In order to reduce the network energy consumption of OTT IM applications effectively, mobile operators need to analyze the contributing factors of high energy consumption arising from users in each quadrant and subsequently address these factors by implementing appropriate solutions.

- Heavy big data users: These users produce or download large amounts of data. Therefore, in this quadrant the service data energy dominates. One of the most effective ways to reduce the network energy consumption of these users is to offload the heavy data services and their users from macro cells to smaller cells (e.g., pico and femto). Several proposals have been made to offload mobile users from macro cells to smaller cells for the purpose of relaxing the capacity of macro cells [28]. However, to date, no research has been conducted to quantify the network energy savings of offloading users to small cells on a per service basis. Furthermore, another major challenge for offloading mobile users from macro cells to small cells is to optimize the overall energy consumption of the mobile 
network, because offloading techniques require a large number of small cells to be activated, which will potentially increase the overall HetNet energy consumption [29]. Therefore, a future research question is how to simultaneously minimize the network energy consumption of mobile services and the overall energy consumption of the mobile network, whilst satisfying the quality-of-experience (QoE).

- Light big data users: For these users, the signaling energy consumption of the mobile network dominates due to the heartbeat mechanism of mobile OTT applications. Intuitively, reducing the heartbeat frequency could reduce the total signaling energy consumption overhead. As the heartbeat mechanism will not only incur excessive waste in network signaling energy but also network resources, existing solutions such as the network socket request manager (NSRM) have been proposed $[15,30]$. Here, instead of allowing every application on user equipment (UE) to send individual heartbeats, the NSRM could bundle the heartbeat requests from all applications in a UE and provide updates at once. However, although this technique reduces the number of heartbeats per UE, it has not considered optimizing the number of heartbeats based on daily user active cycles. As a result, one interesting direction for future work will be to investigate an adaptive control algorithm that dynamically changes the heartbeat request of a UE based on the specific daily user active cycle to further improve the NSRM technique to reduce the signaling overheads per UE.

- Heavy small data users: In an LTE network, traditional applications typically generate traffic only during the connected state. Once the user session is terminated after a certain period of idle time, the UE will transition from the connected state to the idle state. As mentioned in the previous sections, this connecting and releasing process is called RRC, which can cause an excessive amount of signaling overhead [13]. In this quadrant, data traffic arising from each text message is small, so the large signaling overhead generated 
by continuously sending small data packets results in a significant level of energy consumption. For example, referring back to Figure 4, the signaling energy of a text message (regardless of WeChat or SMS) is always much higher than the data energy. The most intuitive way of reducing the SDER is to increase the payload or the amount of data being sent in one RRC cycle, using techniques such as caching and aggregation of data packets. However, the delays introduced by these techniques could potentially affect the QoE of a user. Therefore, a future research question is how to decrease the SDER for heavy small data users while maintaining acceptable QoE.

- Light small data users: The energy consumption caused by the heartbeat mechanism and the signaling overheads generated by sending small data packets dominate the overall energy consumption for users in this quadrant. Combining the potential solutions in the two quadrants: light big data users and heavy small data users, could reduce the overall energy consumption for users in this quadrant. However, the key question here is how to change the behavior of those users who install many applications on their smartphones but do not use them regularly. In this case, our results show that these users would be better off (in terms of network energy efficiency) to use conventional mobile services such as the SMS and MMS. Therefore, to promote the use of conventional services among these users will be a key challenge for mobile operators.

\section{Conclusion}

Ongoing increases in energy consumption of mobile networks due to the rapid deployment of base stations have become a major concern for mobile operators. As current mobile networks are dominated by mobile traffic generated by over-the-top (OTT) mobile applications, it is important for mobile operators to quantify the network energy used to deliver these OTT applications. In this paper, we developed an energy consumption model to assess the network data and signaling energy consumption of mobile services. Since there is a 
current trend in user behavior towards using OTT IM applications over conventional mobile services to send text and voice messages, we found that this change in user behavior could increase network energy consumption for heavy text users. This is due to: (i) network signaling energy overheads consumed by frequent heartbeat signals generated by OTT IM applications; and (ii) network signaling energy overheads consumed by the transmission of small data packets and hint messages. However, we found that for heavy voice message users, WeChat consumes less network energy than conventional voice calls since voice messages are first recorded and then sent in burst packets while conventional voice calls require a dedicated connection throughout the entire call. Furthermore, we defined the signaling to data energy ratio (SDER) metric, which could help mobile operators and OTT IM application developers to evaluate the energy efficiency of mobile services based on different user behaviors and different daily usage patterns. Using this metric together with the findings of this paper, mobile operators and OTT IM application developers could jointly develop energy-efficient techniques and network policies for diverse mobile applications and services with different energy consumption profiles.

\section{References}

1. “China Mobile Limited Annual Report 2014,” China Mobile Limited Annual Report, 2015.

2. World Nuclear Association, Nuclear Power in China, http://www.worldnuclear.org/info/Country-Profiles/Countries-A-F/China--Nuclear-Power/

3. Cisco visual networking index: Forecast and Methodology, 2014-2019, May 2015.

4. GSMA, Mobile's Green Manifesto 2012, http:/www.gsma.com/publicpolicy/wpcontent/uploads/2012/06/Green-Manifesto-2012.pdf 
5. B. G. Molleryd et al., "Analysis of operator options to reduce the impact of the revenue gap caused by flat rate mobile broadband subscriptions," 8th Conf. on Telecom, Media \& Internet Tele-Economics, Jun. 2009.

6. G. Blennerud, "Mobile broadband - busting the myth of the scissor effect," Ericsson business review, pp. 50-55, 2010.

7. G. Auer et al., "How Much Energy Is Needed To Run A Wireless Network?" IEEE Wireless Communications, vol. 18, no. 5, pp. 40-49, Oct. 2011.

8. C. Desset et al., "Flexible power modeling of LTE base stations," 2012 IEEE Wireless Communications and Networking Conference, pp.2858-2862, 2012.

9. Y. K. Chia et al., "Energy cooperation in cellular networks with renewable powered base stations," IEEE Transactions on Wireless Communications, vol. 13, no. 12, pp. 69967010, Dec. 2014.

10. Y. Wu et al., "Green Transmission Technologies for Balancing the Energy Efficiency and Spectrum Efficiency Trade-off," IEEE Communications Magazine, vol. 52, no. 11, pp. 112-120, Nov. 2014.

11. G. Y. Li et al., "Energy-Efficient Wireless Communications: Tutorial, Survey, and Open Issues,” IEEE Wireless Communications, vol. 18, no. 6, pp. 28-35, Dec. 2011.

12. TECHINASIA. WeChat grows to 549M monthly active users, https://www.techinasia.com/wechat-549-million-active-users-q1-2015/

13. M. Gupta et al., "Energy impact of emerging mobile internet applications on LTE networks: issues and solutions," IEEE Communications Magazine, vol. 51, no. 2, pp. 9097, Feb. 2013.

14. Z. Zhang et al., "Study of Signaling Overhead Caused by Keep-Alive Messages in LTE Network,” 2013 IEEE Vehicular Technology Conference, pp. 1-5, Sep. 2013. 
15. Y. Choi et al., "The impact of application signaling traffic on public land mobile networks," IEEE Communications Magazine, vol. 52, no. 1, pp. 166-172, Jan. 2014.

16. Y. Chen et al., "Small data optimized radio access network signaling/control design," 2014 IEEE International Conference on Communications Workshops, pp. 49-54, Jun. 2014.

17. A. Pathak et al., "Where is the energy spent inside my app?: fine grained energy accounting on smartphones with Eprof," 2012 ACM European Conference on Computer Systems, pp. 29-42, Apr. 2012.

18. "Short messaging services versus instant messaging: value versus volume," Deloitte Report, 2014.

19. C. A. Chan et al., "Assessing Network Energy Consumption of Mobile Applications," IEEE Communications Magazine, vol. 53, no. 11, Nov. 2015, pp. 182-191.

20. S. Sesia et al., LTE - The UMTS Long Term Evolution: From Theory to Practice, Wiley, Feb. 2009.

21. "Energy efficiency analysis of the reference systems, areas of improvements and target breakdown," INFSO-ICT-247733 EARTH, 2010.

22. L. Qian et al., "Characterization of 3G control-plane signaling overhead from a dataplane perspective," Proceedings of the 15th ACM international conference on Modeling, analysis and simulation of wireless and mobile systems, pp. 325-332, 2012.

23. GreenTouch Mobile Architecture Doc 1: Models \& Methodology, Version 3.0, Mobile Architecture/Metric Group, Sept. 2012.

24. Ding Li Communications Corp., Ltd. Pilot Pioneer Wireless Network Air Interface Test Tool. http://www.dinglicom.com/products-detail.php?lv1=1\&id=1

25. Y. Luo et al., "Experiment and analysis of WeChat's effects on mobile network," Information and Communications Technology, vol.4, no. 6, pp. 16-20, 2013. 
26. C. I et al., "Toward Green and Soft: A 5G Perspective,” IEEE Communications Magazine, vol. 52, no. 2, pp. 66-73, Feb. 2014.

27. CMCC Hubei report - China Mobile, "China Mobile Hubei network operations report 2013," http://wenku.baidu.com/view/59087826f12d2af90342e61a.html, 2013

28. D. Cao et al., "Optimal combination of base station densities for energy-efficient two-tier heterogeneous cellular networks," IEEE Trans. Wireless Commun., no. 9, vol. 12, pp. 4350-4362, Sept. 2013.

29. J. He et al., "Multi-cell cooperation energy saving solution: An operator's perspective," 2014 IEEE/CIC International Conference on Communications in China (ICCC), pp. 714$717,2014$.

30. “Managing Background Data Traffic in Mobile Devices," Qualcomm document, 2013. 